\title{
Construction of Spirooxindole Skeleton Through Intramolecular Dieckmann Cyclization
}

\author{
Ting Wu $\cdot$ Zhiqiang Pan $\cdot$ Chengfeng Xia
}

Received: 23 March 2017/Accepted: 30 April 2017/Published online: 8 May 2017

(C) The Author(s) 2017. This article is an open access publication

\begin{abstract}
A highly efficient and direct approach was developed to construct the structurally diverse spirooxindole skeleton, which is an important basic motif in natural products. Both the 3,3'-pyrrolidonyl spirooxindoles and spiroindolin-2-one $\delta$ lactones were smoothly obtained by the intramolecular Dieckmann cyclization of oxindoles in excellent yield under mild conditions.
\end{abstract}

Graphical Abstract

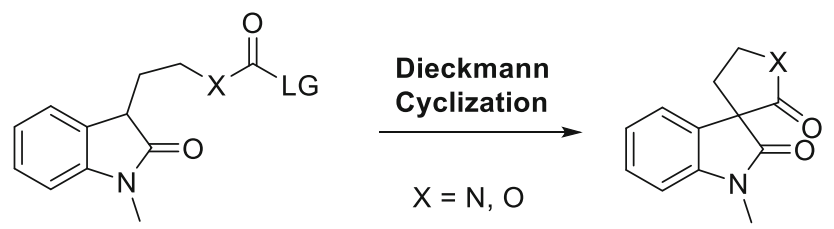

Keywords Dieckmann reaction $\cdot$ Cyclization $\cdot$ Spirooxindole $\cdot$ Indole

\section{Introduction}

The spirooxindole ring system is a popular structure in a number of natural products and leading compounds, which possess significant biological and pharmaceutical activities (Fig. 1). Among them, horsfiline 1 was isolated from the leaves of the Malaysian indigenous tree Horsfieldia superba [1]. Corynoxine $\mathbf{2}$ and corynoxone B $\mathbf{3}$ are the

T. $\mathrm{Wu}$

The Key Laboratory of Chemistry for Natural Products of Guizhou Province, Chinese Academy of Sciences, Guiyang 55002, China

\section{Z. Pan · C. Xia $(\bowtie)$}

Key Laboratory of Medicinal Chemistry for Natural Resources,

Ministry of Education, School of Chemical Science and

Technology, Yunnan University, Kunming 650091, China

e-mail: xiacf@ynu.edu.cn important tetracyclic 3-spirooxindole alkaloids, which were isolated from the hooks of Uncariae plants, and have shown potential applications in the prevention or treatment of Parkinson's disease [2-4]. Other complex spirooxindoles, such as spirotryprostatin B $\mathbf{4}$, was produced by Aspergillus fumigatus and found to inhibit mammalian cancer cell [5]. Cyanogramide 5 was derived from the fermentation broth of the marine-derived Actinoalloteichus cyanogriseus WH1-2216-6 and exhibited strong potencies to reverse the P-glycoprotein-mediated multidrug resistance [6].

The important biological activities and structural complexities of spirooxindole alkaloids have attracted many organic chemists to develop a number of elegant synthetic strategies (Scheme 1). A representative application of the Mannich reaction in complex spirooxindole alkaloid synthesis was documented by Danishefsky in his approach to 


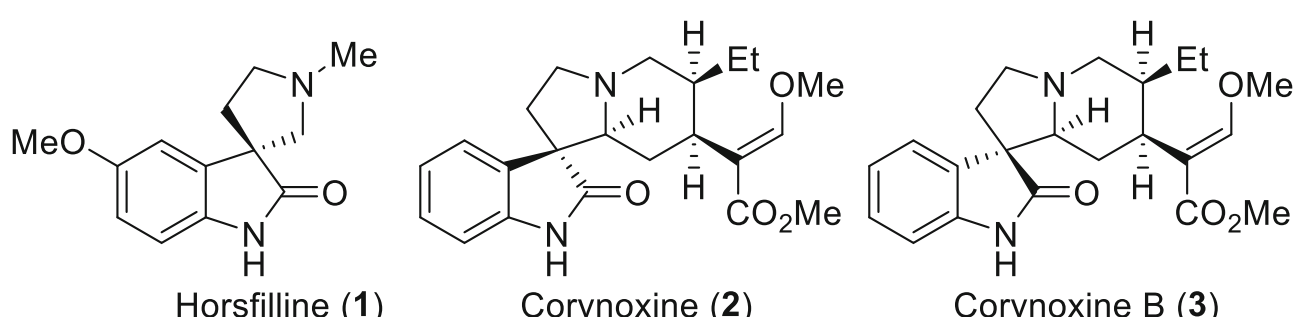

Horsfilline (1)

Corynoxine (2)

Corynoxine B (3)<smiles>CC1=CC(=O)N2C(=O)[C@@]3(C=C2C2CCCN12)C(=O)Nc1ccccc13</smiles>

Spirotryprostatin B (4)<smiles>CO[C@@]1(C)[C@H](/C=C/c2ccccc2)C=c2n3c(c(=O)n21)=CC1(C(=O)N(C)c2ccccc21)C3=O</smiles>

Cyanogramide (5)

Fig. 1 Representative natural products with spirooxindole skeleton<smiles></smiles>

Interruption of the Bischler-Napieralski reaction<smiles>[R]c1ccc(F)cc1</smiles><smiles>[R]C=C1C(=O)N([R2])c2ccccc21</smiles>

Michael addition/cyclization<smiles>CC(C)=C[CH+]C=O</smiles>

Mannich reaction<smiles>[C-]=C</smiles><smiles>C=CC=C</smiles><smiles>C=CC=C</smiles><smiles>[X]CC12CCCC1(CC)C(=O)Nc1ccccc12</smiles><smiles>C=CC=C</smiles><smiles>C=C</smiles>

Oxidative rearrangment<smiles>[R1]C1CCc2c([nH]c3ccccc23)C1</smiles><smiles></smiles>

1,3-Dipole cycloadition<smiles>C=CC=C</smiles><smiles>[R]c1ccc[n+](CCC2C(=O)Nc3ccccc32)c1</smiles>

Cross dehydrogenation

Scheme 1 The general synthetic strategies of spirooxindoles

spirotryprostatin B 4 [7]. The biomimetically oxidative rearrangement of a tetrahydro- $\beta$-carboline was reported to construct the tetracyclic 3-spirooxindole skeleton by Martin [8]. The most widely used method for synthesis of structurally complex spirooxindole structure is the 1,3dipolar cycloaddition (1,3-DC) reaction of azomethine ylide with olefin [9-17]. Moreover, Carreira et al. have developed a reliable methodology to access the pyrrolidinyl-spirooxindole structure via a $\mathrm{MgI}_{2}$-mediated ring- expansion reaction of a spiro[cyclopropane-1,3'"'oxindole] with an aldimine [18]. Other synthetic strategies of the spiro-pyrrolidone-3,3'-oxoindole frameworks also include Michael cyclization reaction [19-23] and C-selective SnAr reactions [24-26]. Besides, in our previous study, a completely different method has been achieved for the construction of the tetracyclic 3-spirooxindole through a transition-metal-free intramolecular cross-dehydrogenative coupling of pyridinium [27]. 
Table 1 Optimization of Dieckmann cyclization<smiles>CN1CCC2(C1=O)C(=O)N(C)c1ccccc12</smiles>

\begin{tabular}{lllll}
\hline Entry & Substrate & $\mathrm{LG}$ & Base & ${\text { Yield }(\%)^{\mathbf{a}}}^{\circ}$ \\
\hline 1 & $\mathbf{6 a}$ & $\mathrm{OMe}$ & LHMDS & 0 \\
2 & $\mathbf{6 a}$ & $\mathrm{OMe}$ & $\mathrm{NaH}$ & 0 \\
3 & $\mathbf{6 a}$ & $\mathrm{OMe}$ & $\mathrm{KHMDS}$ & 0 \\
4 & $\mathbf{6 b}$ & $\mathrm{OPh}$ & $\mathrm{KHMDS}$ & 63 \\
5 & $\mathbf{6 c}$ & $\mathrm{OC}_{6} \mathrm{~F}_{5}$ & $\mathrm{KHMDS}$ & 95 \\
6 & $\mathbf{6 d}$ & $\mathrm{OCH}_{2} \mathrm{CF}_{3}$ & $\mathrm{KHMDS}$ & 83 \\
7 & $\mathbf{6 e}$ & $\mathrm{OPhNO}_{2}$ & $\mathrm{KHMDS}$ & Quant. \\
8 & $\mathbf{6 e}$ & $\mathrm{OPhNO}_{2}$ & $\mathrm{NaHMDS}$ & Quant. \\
9 & $\mathbf{6 e}$ & $\mathrm{OPhNO}_{2}$ & $\mathrm{LHMDS}$ & Quant. \\
10 & $\mathbf{6 e}$ & $\mathrm{OPhNO}_{2}$ & $\mathrm{LDA}$ & 85 \\
11 & $\mathbf{6 e}$ & $\mathrm{OPhNO}_{2}$ & $\mathrm{NaH}$ & 63 \\
12 & $\mathbf{6 e}$ & $\mathrm{OPhNO}_{2}$ & $t$-BuOK & 0 \\
13 & $\mathbf{6 e}$ & $\mathrm{OPhNO}_{2}$ & $\mathrm{Et}_{3} \mathrm{~N}$ & 0 \\
14 & $\mathbf{6 e}$ & $\mathrm{OPhNO}_{2}$ & $\mathrm{~K}_{2} \mathrm{CO}$ & 3 \\
\hline
\end{tabular}

${ }^{\text {a }}$ Isolated yields by silica gel column

Although many elegant methods have been developed for the construction of spirooxindole skeletons, strategies that could directly and efficiently obtain the structurally diverse spirooxindoles are still limited. Therefore, searching for new approach for the construction of spirooxindole is still required. As a well-known organic reaction, Dieckmann cyclization was widely used in the preparation of natural products and medicines, but with limited reports on the construction of natural alkaloid skeletons [28-32]. In 2012, Zhang reported that bromoanilides underwent the Dieckmann condensation in the presence of copper (I) iodide and LHMDS to give the spirooxindoles [33]. In this work, we have developed a highly efficient and direct method to construct the spirooxindoles skeleton by the intramolecular Dieckmann cyclization.

\section{Results and Discussion}

The starting 2-oxytryptamines 6 were easily prepared by direct oxidation of the corresponding tryptamine or tryptophol under modified conditions [34, 35]. At the outset, 2-oxytryptamine methoxyl carbamate 6a was employed to screen the construction of spirooxindole by intramolecular Dieckmann cyclization. Unfortunately, no cyclized product was detected under various conditions (Table 1, entries 1-3). We speculated that the Dieckmann cyclization proceeded with the removal of methoxyl group during the reaction. However, the methoxyl group was a very weak leaving group, thus inhibited the cyclization process. To facilitate the cyclization, phenyl carbamate substrate $\mathbf{6 b}$ was subjected to the reaction. As a result, the spirooxindole 7 was obtained in acceptable yield when KHMDS was used as base (Table 1, entry 4). Shift the leaving group from phenol to pentafluorophenol afforded the spirooxindole 7 excellent yield. Instead, when trifluoroethanol was used as leaving group, the generation of spirooxindole 7 was among the phenol and pentafluorophenol. We discovered that the best leaving group for the Dieckmann cyclization was $p$-nitrophenol and almost quantitative yield was achieved (Table 1, entry 7). Changing the base from KHMDS to NaHMDS or LHMDS also afforded excellent yields of cyclization (Table 1, entries 8 and 9). However, other bases, such as LDA, NaH, and $t$ $\mathrm{BuOK}$, resulted in lower yields. When the weak bases $\left(\mathrm{Et}_{3} \mathrm{~N}\right.$ or $\mathrm{K}_{2} \mathrm{CO}_{3}$ ) were subjected, no spirooxindole product was generated (Table 1, entries 13 and 14).

With the established optimal conditions, different $p$-nitrophenol carbamates were conducted to investigate the scope of Dieckmann cyclization. It was found that the electron-withdrawing substitutions at C-5 position of 
Table 2 Substrate scope of the Dieckmann cyclization<smiles>[X]C1=C(C)C2(CCC2)C(=O)N([R])c2ccccc21</smiles><smiles>COc1ccc2c(c1)C1(CCN(C)C1=O)C(=O)N2C</smiles>

8, $92 \%$<smiles>CN1CCC2(C1=O)C(=O)N(C)c1ccc(F)cc12</smiles>

$11,81 \%$<smiles>C=CCN1CCC2(C1=O)C(=O)N(C)c1ccccc12</smiles>

$14,60 \%$<smiles>CN1C(=O)C2(CCOC2=O)c2ccccc21</smiles>

$17,58 \%$<smiles>Cc1ccc2c(c1)C1(CCN(C)C1=O)C(=O)N2C</smiles>

9, $89 \%$<smiles>C=CCN1C(=O)C2(CCN(C)C2=O)c2ccccc21</smiles>

$12,70 \%$<smiles>CN1C(=O)C2(CCN(Cc3ccccc3)C2=O)c2ccccc21</smiles>

$15,75 \%$<smiles>CN1C(=O)C2(CCOC2=O)c2cc(Br)ccc21</smiles>

$18,30 \%$<smiles>CN1CCC2(C1=O)C(=O)N(C)c1ccc(Cl)cc12</smiles>

$10,85 \%$<smiles>CN1CCC2(C1=O)C(=O)N(Cc1ccccc1)c1ccccc12</smiles>

$13,80 \%$<smiles>CN1CCC2(C(=O)Nc3ccccc32)C1=O</smiles>

$16,43 \%$<smiles>CN1C(=O)C2(CCOC2=O)c2cc(Cl)ccc21</smiles>

$19,43 \%$ tryptamine oxindoles gave lower yields (Table 2, compounds 10 and 11). We reasoned that it might be due to the lower reactivities of the tryptamine oxindoles as nucleophile to attack the $p$-nitrophenol carbamates. When both of the N1 and $\mathrm{N}$-terminal were substituted with different alkyl groups, the Dieckmann cyclization proceeded smoothly with good yields (Table 2, compounds 12-15). Notably, when there was no protecting group at the $\mathrm{N} 1$ position of tryptamine oxindole, the cyclization also gave the target product in reasonable yield. This indicated that the $\mathrm{C}-3$ hydrogen was earlier to be deprotonated than the hydrogen of N1. Besides the $p$-nitrophenol carbamate of tryptamine oxindoles, the $p$ nitrophenol carbonate of oxytryptophols were also examined for the Dieckmann cyclization. Although the yields were lower than carbamates, the corresponding spiroindolin-2-one $\delta$-lactones $\mathbf{1 9}$ and $\mathbf{2 0}$ were obtained with acceptable yields. 
In conclusion, we have developed a highly efficient and direct strategy for the construction of 3,3'-pyrrolidonyl spirooxindoles and spiroindolin-2-one $\delta$-lactones under mild conditions. The reactions were achieved through an intramolecular Dieckmann cyclization of oxytrytamine carbamates to provide a series of spirooxindole derivatives in high yield.

\section{Experimental Section}

\subsection{General Procedures for Synthesis of Spirooxindole}

KHMDS (0.18 mmol) was added dropwise to a solution of indolin-2-one $6(0.12 \mathrm{mmol})$ in anhydrous THF $(5 \mathrm{~mL})$ at $0{ }^{\circ} \mathrm{C}$. After stirring for $30 \mathrm{~min}$, the reaction temperature was allowed to warm to room temperature and continued stirring for additional $2 \mathrm{~h}$. The reaction was quenched by saturated aqueous $\mathrm{NH}_{4} \mathrm{Cl}$ and extracted by $\mathrm{CH}_{2} \mathrm{Cl}_{2}$ $(5 \mathrm{~mL} \times 3)$. The combined organic layers was washed with brine, dried over anhydrous $\mathrm{Na}_{2} \mathrm{SO}_{4}$ and concerned. The residue was purified by silica gel column $\left(\mathrm{CH}_{2} \mathrm{Cl}_{2} /\right.$ acetone, 2:1) to afford the spirooxindole.

7. White powder $\left(63 \%\right.$ yield). ${ }^{1} \mathrm{H}$ NMR $(400 \mathrm{MHz}$, $\left.\mathrm{CDCl}_{3}\right) \delta: 7.29$ (t, $\left.J=7.8 \mathrm{~Hz}, 1 \mathrm{H}\right), 7.14(\mathrm{~d}, J=7.5 \mathrm{~Hz}$ $1 \mathrm{H}), 7.06$ (t, $J=7.5 \mathrm{~Hz}, 1 \mathrm{H}), 6.86(\mathrm{~d}, J=7.8 \mathrm{~Hz}, 1 \mathrm{H})$, $3.78(\mathrm{td}, J=8.8,6.3 \mathrm{~Hz}, 1 \mathrm{H}), 3.59(\mathrm{td}, J=9.0,4.6 \mathrm{~Hz}$, $1 \mathrm{H}), 3.21$ (s, 3H), 2.97 (s, 3H), 2.67 (ddd, $J=13.0,8.3$, $4.6 \mathrm{~Hz}, 1 \mathrm{H}), 2.38(\mathrm{ddd}, J=13.3,8.5,6.2 \mathrm{~Hz}, 1 \mathrm{H}) ;{ }^{13} \mathrm{C}$ NMR $\left(100 \mathrm{MHz}, \mathrm{CDCl}_{3}\right) \delta: 175.7,170.5,144.5,129.9$, 129.0, 123.1, 122.8, 108.6, 57.8, 47.2, 30.5, 29.5, 26.6; HR-ESI-MS $(\mathrm{m} / \mathrm{z})$ : calcd. for $\mathrm{C}_{13} \mathrm{H}_{15} \mathrm{~N}_{2} \mathrm{O}_{2}[\mathrm{M}+\mathrm{H}]^{+}$, 231.1128, found 231.1130.

8. White powder $\left(92 \%\right.$ yield); ${ }^{1} \mathrm{H}$ NMR $(400 \mathrm{MHz}$, $\left.\mathrm{CDCl}_{3}\right) \delta: 6.75(\mathrm{dd}, J=8.4,2.1 \mathrm{~Hz}, 1 \mathrm{H}), 6.69-6.67(\mathrm{~m}$, $2 \mathrm{H}), 3.74-3.68(\mathrm{~m}, 4 \mathrm{H}), 3.50(\mathrm{td}, J=9.0,4.5 \mathrm{~Hz}, 1 \mathrm{H})$, $3.12(\mathrm{~s}, 3 \mathrm{H}), 2.90$ (s, 3H), 2.60 (ddd, $J=12.9,8.2,4.5 \mathrm{~Hz}$, 1H), $2.45-2.18(\mathrm{~m}, 1 \mathrm{H}) .{ }^{13} \mathrm{C} \mathrm{NMR}\left(100 \mathrm{MHz}, \mathrm{CDCl}_{3}\right) \delta$ : $174.3,169.4,155.3,136.9,130.0,112.1,109.5,107.8$, 57.1, 54.8, 46.1, 29.5, 28.5, 25.6; HR-ESI-MS (m/z): calcd. for $\mathrm{C}_{13} \mathrm{H}_{15} \mathrm{~N}_{2} \mathrm{O}_{2}[\mathrm{M}+\mathrm{H}]^{+}, \quad 261.1234$, found 261.1235 .

9. White powder ( $89 \%$ yield); ${ }^{1} \mathrm{H}$ NMR $(400 \mathrm{MHz}$, $\left.\mathrm{CDCl}_{3}\right) \delta: 7.09(\mathrm{~d}, J=7.9 \mathrm{~Hz}, 1 \mathrm{H}), 6.96(\mathrm{~s}, 1 \mathrm{H}), 6.73(\mathrm{~d}$, $J=7.9 \mathrm{~Hz}, 1 \mathrm{H}), 3.76(\mathrm{td}, J=8.7,6.4 \mathrm{~Hz}, 1 \mathrm{H}), 3.57(\mathrm{td}$, $J=9.0,4.6 \mathrm{~Hz}, 1 \mathrm{H}), 3.18$ (s, 3H), 2.96 (s, 3H), 2.65 (ddd, $J=13.0,8.3,4.6 \mathrm{~Hz}, 1 \mathrm{H}), 2.39-2.32(\mathrm{~m}, 1 \mathrm{H}), 2.30(\mathrm{~s}$, $3 \mathrm{H}) ;{ }^{13} \mathrm{C}$ NMR $\left(100 \mathrm{MHz}, \mathrm{CDCl}_{3}\right) \delta: 175.3,170.4,141.8$, $132.3,129.6,128.9,123.3,107.9,57.5,46.9,30.2,29.2$, 26.3, 20.8; HR-ESI-MS (m/z): calcd. for $\mathrm{C}_{14} \mathrm{H}_{17} \mathrm{~N}_{2} \mathrm{O}_{2}$ $[\mathrm{M}+\mathrm{H}]^{+}, 245.1285$, found 245.1286 .

10. White powder $\left(85 \%\right.$ yield); ${ }^{1} \mathrm{H}$ NMR $(400 \mathrm{MHz}$, $\left.\mathrm{CDCl}_{3}\right) \delta: 7.27-7.14(\mathrm{~m}, 1 \mathrm{H}), 7.12(\mathrm{~d}, J=1.6 \mathrm{~Hz}, 1 \mathrm{H})$, $6.77(\mathrm{~d}, J=8.3 \mathrm{~Hz}, 1 \mathrm{H}), 3.79(\mathrm{dd}, J=15.4,8.7 \mathrm{~Hz}, 1 \mathrm{H})$, $3.57(\mathrm{td}, J=9.1,4.5 \mathrm{~Hz}, 1 \mathrm{H}), 3.19(\mathrm{~s}, 3 \mathrm{H}), 2.97(\mathrm{~s}, 3 \mathrm{H})$, 2.67 (ddd, $J=13.0,8.3,4.5 \mathrm{~Hz}, 1 \mathrm{H}), 2.40-2.33(\mathrm{~m}, 1 \mathrm{H})$; ${ }^{13} \mathrm{C}$ NMR $\left(100 \mathrm{MHz}, \mathrm{CDCl}_{3}\right) \delta: 174.2,168.8,142.1,130.4$, 127.9, 127.3, 122.4, 108.4, 56.8, 46.1, 29.5, 28.3, 25.7; HRESI-MS $(\mathrm{m} / \mathrm{z})$ : calcd. for $\mathrm{C}_{13} \mathrm{H}_{14} \mathrm{ClN}_{2} \mathrm{O}_{2}[\mathrm{M}+\mathrm{H}]^{+}$, 265.0738, found 265.0738.

11. White powder $\left(81 \%\right.$ yield); ${ }^{1} \mathrm{H}$ NMR $(400 \mathrm{MHz}$, $\left.\mathrm{CDCl}_{3}\right) \delta: 7.08(\mathrm{dd}, J=8.1,5.2 \mathrm{~Hz}, 1 \mathrm{H}), 6.86-6.69(\mathrm{~m}$, $1 \mathrm{H}), 6.60(\mathrm{dd}, J=8.8,2.1 \mathrm{~Hz}, 1 \mathrm{H}), 3.84-3.72(\mathrm{~m}, 1 \mathrm{H})$, $3.56(\mathrm{td}, J=9.1,4.3 \mathrm{~Hz}, 1 \mathrm{H}), 3.19(\mathrm{~s}, 3 \mathrm{H}), 2.97(\mathrm{~s}, 3 \mathrm{H})$, 2.65 (ddd, $J=12.8,8.2,4.3 \mathrm{~Hz}, 1 \mathrm{H}), 2.36$ (ddd, $J=13.3$, 8.6, $6.6 \mathrm{~Hz}, 1 \mathrm{H}) ;{ }^{13} \mathrm{C}$ NMR $\left(100 \mathrm{MHz}, \mathrm{CDCl}_{3}\right) \delta: 174.9$, $169.1,162.5(\mathrm{~d}, J=245 \mathrm{~Hz}), 145.1(\mathrm{~d}, J=11 \mathrm{~Hz}), 123.5$ $(\mathrm{d}, J=118 \mathrm{~Hz}), 122.9,108.0(\mathrm{~d}, J=23 \mathrm{~Hz}), 96.5(\mathrm{~d}$, $J=48 \mathrm{~Hz}), 56.3,46.0,29.5,28.4,25.7$; HR-ESI-MS (m/ z): calcd. for $\mathrm{C}_{13} \mathrm{H}_{14} \mathrm{FN}_{2} \mathrm{O}_{2}[\mathrm{M}+\mathrm{H}]^{+}, 249.1034$, found 249.1033 .

12. White powder $\left(70 \%\right.$ yield); ${ }^{1} \mathrm{H}$ NMR $(400 \mathrm{MHz}$, $\left.\mathrm{CDCl}_{3}\right) \delta: 7.15(\mathrm{t}, J=7.8 \mathrm{~Hz}, 1 \mathrm{H}), 7.05(\mathrm{~d}, J=7.1 \mathrm{~Hz}$, $1 \mathrm{H}), 6.95(\mathrm{t}, J=7.5 \mathrm{~Hz}, 1 \mathrm{H}), 6.73(\mathrm{~d}, J=7.8 \mathrm{~Hz}, 1 \mathrm{H})$, $5.85-5.60(\mathrm{~m}, 1 \mathrm{H}), 5.14(\mathrm{dd}, J=28.1,13.8 \mathrm{~Hz}, 2 \mathrm{H})$, $4.34-4.12(\mathrm{~m}, 2 \mathrm{H}), 3.68(\mathrm{td}, J=8.7,6.4 \mathrm{~Hz}, 1 \mathrm{H}), 3.48(\mathrm{td}$, $J=9.0,4.6 \mathrm{~Hz}, 1 \mathrm{H}), 2.87(\mathrm{~s}, 3 \mathrm{H}), 2.59(\mathrm{ddd}, J=13.0$, $8.3,4.6 \mathrm{~Hz}, 1 \mathrm{H}), 2.30(\mathrm{ddd}, J=13.3,8.6,6.3 \mathrm{~Hz}, 1 \mathrm{H})$; ${ }^{13} \mathrm{C}$ NMR $\left(100 \mathrm{MHz}, \mathrm{CDCl}_{3}\right) \delta: 175.5,170.4,143.6,130.7$, 129.9, 128.8, 122.9, 122.8, 117.5, 109.3, 57.8, 47.2, 42.4, 30.5, 29.3; HR-ESI-MS (m/z): calcd. for $\mathrm{C}_{15} \mathrm{H}_{17} \mathrm{~N}_{2} \mathrm{O}_{2}$ $[\mathrm{M}+\mathrm{H}]^{+}, 257.1285$, found 257.1288 .

13. White powder $\left(80 \%\right.$ yield); ${ }^{1} \mathrm{H}$ NMR $(400 \mathrm{MHz}$, $\left.\mathrm{CDCl}_{3}\right) \delta: 7.31-7.10(\mathrm{~m}, 5 \mathrm{H}), 7.10-6.98(\mathrm{~m}, 2 \mathrm{H}), 6.92(\mathrm{t}$, $J=7.5 \mathrm{~Hz}, 1 \mathrm{H}), 6.68-6.49(\mathrm{~m}, 1 \mathrm{H}), 4.91(\mathrm{~d}, J=16.0 \mathrm{~Hz}$, $1 \mathrm{H}), 4.73(\mathrm{~d}, J=16.0 \mathrm{~Hz}, 1 \mathrm{H}), 3.70(\mathrm{dd}, J=15.1$, $8.8 \mathrm{~Hz}, 1 \mathrm{H}), 3.51(\mathrm{td}, J=9.0,4.7 \mathrm{~Hz}, 1 \mathrm{H}), 2.88(\mathrm{~s}, 3 \mathrm{H})$, 2.64 (ddd, $J=13.1,8.4,4.7 \mathrm{~Hz}, 1 \mathrm{H}), 2.40-2.26(\mathrm{~m}, 1 \mathrm{H})$; ${ }^{13} \mathrm{C}$ NMR $\left(100 \mathrm{MHz}, \mathrm{CDCl}_{3}\right) \delta: 175.9,170.5,143.55$, $135.3,130.0,128.9,128.8,127.5,127.0,123.0,122.8$, 109.5, 57.8, 47.2, 43.9, 30.5, 29.4; HR-ESI-MS (m/z): calcd. for $\mathrm{C}_{19} \mathrm{H}_{19} \mathrm{~N}_{2} \mathrm{O}_{2}[\mathrm{M}+\mathrm{H}]^{+}, \quad 307.1441$, found 307.1443 .

14. White powder $\left(60 \%\right.$ yield); ${ }^{1} \mathrm{H}$ NMR $(400 \mathrm{MHz}$, $\left.\mathrm{CDCl}_{3}\right) \delta: 7.31(\mathrm{~d}, J=10.3 \mathrm{~Hz}, 1 \mathrm{H}), 7.16(\mathrm{~d}, J=7.1 \mathrm{~Hz}$, $1 \mathrm{H}), 7.07(\mathrm{t}, J=7.5 \mathrm{~Hz}, 1 \mathrm{H}), 6.86(\mathrm{~d}, J=7.8 \mathrm{~Hz}, 1 \mathrm{H})$, 5.80 (ddt, $J=16.2,10.3,5.9 \mathrm{~Hz}, 1 \mathrm{H}), 5.44-5.19(\mathrm{~m}, 2 \mathrm{H})$, $4.00(\mathrm{dd}, J=3.8,1.9 \mathrm{~Hz}, 2 \mathrm{H}), 3.84-3.67(\mathrm{~m}, 1 \mathrm{H}), 3.55$ $(\mathrm{td}, J=9.0,4.6 \mathrm{~Hz}, 1 \mathrm{H}), 3.22(\mathrm{~s}, 3 \mathrm{H}), 2.68$ (ddd, $J=12.9,8.2,4.5 \mathrm{~Hz}, 1 \mathrm{H}), 2.39$ (ddd, $J=13.2,8.5$, $6.4 \mathrm{~Hz}, 1 \mathrm{H}) .{ }^{13} \mathrm{C}$ NMR $\left(100 \mathrm{MHz}, \mathrm{CDCl}_{3}\right) \delta: 175.6,170.3$, $144.5,131.9,129.8,129.0,123.0,122.7,118.3,108.5$, 58.0, 46.0, 44.6, 29.5, 26.5; HR-ESI-MS (m/z): calcd. for $\mathrm{C}_{5} \mathrm{H}_{17} \mathrm{~N}_{2} \mathrm{O}_{2}[\mathrm{M}+\mathrm{H}]^{+}, 257.1285$, found 257.1286.

15. White powder $\left(75 \%\right.$ yield); ${ }^{1} \mathrm{H}$ NMR $(400 \mathrm{MHz}$, $\left.\mathrm{CDCl}_{3}\right) \delta: 7.41-7.27(\mathrm{~m}, 6 \mathrm{H}), 7.18-7.03(\mathrm{~m}, 2 \mathrm{H}), 6.87(\mathrm{~d}$, 
$J=7.8 \mathrm{~Hz}, 1 \mathrm{H}), 4.64(\mathrm{~d}, J=16.0 \mathrm{~Hz}, 1 \mathrm{H}), 4.50(\mathrm{~d}$, $J=16.0 \mathrm{~Hz}, 1 \mathrm{H}), 3.67(\mathrm{td}, J=8.9,6.1 \mathrm{~Hz}, 1 \mathrm{H}), 3.44(\mathrm{td}$, $J=9.0,4.9 \mathrm{~Hz}, 1 \mathrm{H}), 3.24(\mathrm{~s}, 3 \mathrm{H}), 2.66(\mathrm{ddd}, J=13.2$, 8.4, $4.9 \mathrm{~Hz}, 1 \mathrm{H}), 2.56-2.24(\mathrm{~m}, 1 \mathrm{H}) ;{ }^{13} \mathrm{C}$ NMR $(100 \mathrm{MHz}$, $\left.\mathrm{CDCl}_{3}\right) \delta: 175.5,170.68,144.5,135.9,129.9,129.1,128.8$, $128.1,127.7,123.1,122.7,108.6,57.9,47.4,44.5,29.4$, 26.6; HR-ESI-MS (m/z): calcd. for $\mathrm{C}_{19} \mathrm{H}_{19} \mathrm{~N}_{2} \mathrm{O}_{2}$ $[\mathrm{M}+\mathrm{H}]^{+}, 307.1441,307.1442$.

16. White powder ( $43 \%$ yield); ${ }^{1} \mathrm{H}$ NMR $(400 \mathrm{MHz}$, $\left.\mathrm{CDCl}_{3}\right) \delta: 8.17$ (brs, $\left.1 \mathrm{H}\right), 7.20(\mathrm{t}, J=7.7 \mathrm{~Hz}, 1 \mathrm{H}), 7.13(\mathrm{~d}$, $J=7.3 \mathrm{~Hz}, 1 \mathrm{H}), 7.02(\mathrm{t}, J=7.5 \mathrm{~Hz}, 1 \mathrm{H}), 6.84(\mathrm{~d}$, $J=7.8 \mathrm{~Hz}, 1 \mathrm{H}), 3.77(\mathrm{td}, J=8.9,6.1 \mathrm{~Hz}, 1 \mathrm{H}), 3.60(\mathrm{td}$, $J=9.0,4.8 \mathrm{~Hz}, 1 \mathrm{H}), 2.99(\mathrm{~s}, 3 \mathrm{H}), 2.72(\mathrm{ddd}, J=13.2$, 8.4, 4.8 Hz, 1H), 2.56-2.29 (m, 1H); ${ }^{13} \mathrm{C}$ NMR (100 MHz, $\left.\mathrm{CDCl}_{3}\right) \delta: 177.3,170.5,141.6,130.4,128.9,123.1,122.9$, 110.2, 58.2, 47.3, 30.6, 29.4; HR-ESI-MS (m/z): calcd. for $\mathrm{C}_{12} \mathrm{H}_{12} \mathrm{~N}_{2} \mathrm{O}_{2}[\mathrm{M}+\mathrm{H}]^{+}, 217.0972$, found 217.0974.

17. White powder (58\% yield); ${ }^{1} \mathrm{H}$ NMR $(400 \mathrm{MHz}$, $\left.\mathrm{CDCl}_{3}\right) \delta: 7.37(\mathrm{t}, J=7.7 \mathrm{~Hz}, 1 \mathrm{H}), 7.23(\mathrm{~d}, J=7.3 \mathrm{~Hz}$, $1 \mathrm{H}), 7.12(\mathrm{t}, J=7.5 \mathrm{~Hz}, 1 \mathrm{H}), 6.90(\mathrm{~d}, J=7.8 \mathrm{~Hz}, 1 \mathrm{H})$, $4.62(\mathrm{td}, J=8.5,5.0 \mathrm{~Hz}, 1 \mathrm{H}), 4.62(\mathrm{td}, J=8.5,5.0 \mathrm{~Hz}$, $1 \mathrm{H}), 3.25(\mathrm{~s}, 3 \mathrm{H}), 2.91$ (ddd, $J=12.7,7.4,5.0 \mathrm{~Hz}, 1 \mathrm{H})$, $2.67(\mathrm{dt}, J=13.2,7.9 \mathrm{~Hz}, 1 \mathrm{H}) ;{ }^{13} \mathrm{C}$ NMR $(100 \mathrm{MHz}$, $\left.\mathrm{CDCl}_{3}\right) \delta: 173.3,172.9,144.4,129.7,127.7,123.5,123.0$, 108.9, 66.7, 55.6, 33.5, 26.7; HR-ESI-MS (m/z): calcd. for $\mathrm{C}_{12} \mathrm{H}_{12} \mathrm{NO}_{3}[\mathrm{M}+\mathrm{H}]^{+}, 218.0812$, found 218.0809.

18. White powder (30\% yield); ${ }^{1} \mathrm{H}$ NMR $(400 \mathrm{MHz}$, $\left.\mathrm{CDCl}_{3}\right) \delta: 7.49(\mathrm{dd}, J=8.3,1.6 \mathrm{~Hz}, 1 \mathrm{H}), 7.34(\mathrm{~d}$, $J=1.5 \mathrm{~Hz}, 1 \mathrm{H}), 6.77(\mathrm{~d}, J=8.3 \mathrm{~Hz}, 1 \mathrm{H}), 4.81 \quad(\mathrm{q}$, $J=7.9 \mathrm{~Hz}, 1 \mathrm{H}), 4.60(\mathrm{td}, J=8.6,4.7 \mathrm{~Hz}, 1 \mathrm{H}), 3.23(\mathrm{~d}$, $J=8.3 \mathrm{~Hz}, 3 \mathrm{H}$ ), 2.89 (ddd, $J=12.5,7.5,4.7 \mathrm{~Hz}, 1 \mathrm{H}$ ), $2.66(\mathrm{dt}, J=13.2,8.1 \mathrm{~Hz}, 1 \mathrm{H}) ;{ }^{13} \mathrm{C}$ NMR $(100 \mathrm{MHz}$, $\left.\mathrm{CDCl}_{3}\right) \delta: 172.7,172.2,143.5,132.6,129.5,126.4,115.9$, 110.3, 66.8, 55.6, 33.4, 26.8; HR-ESI-MS (m/z): calcd. for $\mathrm{C}_{12} \mathrm{H}_{10} \mathrm{NBrO}_{3} \mathrm{~K}[\mathrm{M}+\mathrm{K}]^{+}, 333.9476$, found 333.9484 .

19. White powder (43\% yield); ${ }^{1} \mathrm{H}$ NMR $(400 \mathrm{MHz}$, $\left.\mathrm{CDCl}_{3}\right) \delta: 6.88(\mathrm{dd}, J=8.4,2.2 \mathrm{~Hz}, 1 \mathrm{H}), 6.83(\mathrm{~d}$, $J=2.1 \mathrm{~Hz}, 1 \mathrm{H}), 6.80(\mathrm{~d}, J=8.5 \mathrm{~Hz}, 1 \mathrm{H}), 4.82(\mathrm{q}$, $J=7.9 \mathrm{~Hz}, 1 \mathrm{H}), 4.60(\mathrm{td}, J=8.5,5.0 \mathrm{~Hz}, 1 \mathrm{H}), 3.79(\mathrm{~s}$, $3 \mathrm{H}), 3.22(\mathrm{~s}, 3 \mathrm{H}), 2.90(\mathrm{ddd}, J=12.5,7.5,4.7 \mathrm{~Hz}, 1 \mathrm{H})$, $2.66(\mathrm{dt}, J=13.2,8.1 \mathrm{~Hz}, 1 \mathrm{H}) ;{ }^{13} \mathrm{C}$ NMR $(100 \mathrm{MHz}$, $\left.\mathrm{CDCl}_{3}\right) \delta: 172.9,172.9,156.6,137.8,128.8,113.9,110.6$, 109.3, 66.7, 55.9, 55.9, 33.6, 26.8; HR-ESI-MS (m/z): calcd. for $\mathrm{C}_{13} \mathrm{H}_{14} \mathrm{NO}_{4}[\mathrm{M}+\mathrm{H}]^{+}, \quad 248.0917$, found 248.0916 .

Acknowledgements This work was financially supported by the Natural Science Foundation of Yunnan Province (2014FA008 and 2015FB167), and the Program for Changjiang Scholars and Innovative Research Team in University (IRT13095).

\section{Compliance with Ethical Standards}

Conflict of interest The authors declare no conflict of interest.
Open Access This article is distributed under the terms of the Creative Commons Attribution 4.0 International License (http:// creativecommons.org/licenses/by/4.0/), which permits unrestricted use, distribution, and reproduction in any medium, provided you give appropriate credit to the original author(s) and the source, provide a link to the Creative Commons license, and indicate if changes were made.

\section{References}

1. A. Jossang, P. Jossang, H.A. Hadi, T. Sevenet, B. Bodo, J. Org. Chem. 56, 6527 (1991)

2. K. Wang, X.Y. Zhou, Y.Y. Wang, M.M. Li, Y.S. Li, L.Y. Peng, X. Cheng, Y. Li, Y.P. Wang, Q.S. Zhao, J. Nat. Prod. 74, 12 (2011)

3. J.X. Song, J.H. Lu, L.F. Liu, L.L. Chen, S.S.K. Durairajan, Z. Yue, H.Q. Zhang, M. Li, Autophagy 10, 144 (2014)

4. I. Sakakibara, H. Takahashi, S. Terabayashi, M. Yuzurihara, M. Kubo, A. Ishige, M. Higuchi, Y. Komatsu, M. Okada, M. Maruno, C. Biqiang, H.X. Jiang, Phytomedicine 5, 83 (1998)

5. C.B. Cui, H. Kakeya, H. Osada, Tetrahedron 52, 12651 (1996)

6. P. Fu, F. Kong, X. Li, Y. Wang, W. Zhu, Org. Lett. 16, 3708 (2014)

7. F. von Nussbaum, S.J. Danishefsky, Angew. Chem. Int. Ed. 39, $2175(2000)$

8. A. Deiters, M. Pettersson, S.F. Martin, J. Org. Chem. 71, 6547 (2006)

9. J.N.S. Rao, R. Raghunathan, Tetrahedron Lett. 56, 1539 (2015)

10. J.N.S. Rao, R. Raghunathan, Tetrahedron Lett. 53, 854 (2012)

11. J.N.S. Rao, R. Raghunathan, Tetrahedron Lett. 54, 6568 (2013)

12. A. Awata, T. Arai, Chem. Eur. J. 18, 8278 (2012)

13. D. Cheng, Y. Ishihara, B. Tan, C.F. Barbas, ACS Catal. 4, 743 (2014)

14. B.M. Trost, N. Cramer, S.M. Silverman, J. Am. Chem. Soc. 129, 12396 (2007)

15. P.R. Sebahar, R.M. Williams, J. Am. Chem. Soc. 122, 5666 (2000)

16. B. Tan, N.R. Candeias, C.F. Barbas, J. Am. Chem. Soc. 133, 4672 (2011)

17. L. Wang, D. Yang, D. Li, X. Liu, Q. Zhao, R. Zhu, B. Zhang, R. Wang, Org. Lett. 17, 4260 (2015)

18. P.B. Alper, C. Meyers, A. Lerchner, D.R. Siegel, E.M. Carreira, Angew. Chem. Int. Ed. 38, 3186 (1999)

19. Y. Cao, X. Jiang, L. Liu, F. Shen, F. Zhang, R. Wang, Angew. Chem. Int. Ed. 50, 9124 (2011)

20. B.D. Cui, J. Zuo, J.Q. Zhao, M.Q. Zhou, Z.J. Wu, X.M. Zhang, W.C. Yuan, J. Org. Chem. 79, 5305 (2014)

21. L. Chen, Z.J. Wu, M.L. Zhang, D.F. Yue, X.M. Zhang, X.Y. Xu, W.C. Yuan, J. Org. Chem. 80, 12668 (2015)

22. X.L. Liu, W.Y. Han, X.M. Zhang, W.C. Yuan, Org. Lett. 15, 1246 (2013)

23. J.Q. Zhao, M.Q. Zhou, Z.J. Wu, Z.H. Wang, D.F. Yue, X.Y. Xu, X.M. Zhang, W.C. Yuan, Org. Lett. 17, 2238 (2015)

24. S. Sen, V.R. Potti, R. Surakanti, Y.L.N. Murthy, R. Pallepogu, Org. Biomol. Chem. 9, 358 (2011)

25. S. Kobbelgaard, M. Bella, K.A. Jørgensen, J. Org. Chem. 71, 4980 (2006)

26. M. Bella, S. Kobbelgaard, K.A. Jørgensen, J. Am. Chem. Soc. 127, 3670 (2005)

27. J. Xu, L.D. Shao, D. Li, X. Deng, Y.C. Liu, Q.S. Zhao, C. Xia, J. Am. Chem. Soc. 136, 17962 (2014)

28. X. Wang, D. Xia, L. Tan, H. Chen, H. Huang, H. Song, Y. Qin, Chem. Eur. J. 21, 14602 (2015)

29. E.V. Mercado-Marin, R. Sarpong, Chem. Sci. 6, 5048 (2015) 
30. J. Peng, D.L.J. Clive, J. Org. Chem. 74, 513 (2009)

31. C.L. Aboussafy, D.L.J. Clive, J. Org. Chem. 77, 5125 (2012)

32. I. Mutule, B. Joo, Z. Medne, T. Kalnins, E. Vedejs, E. Suna, J. Org. Chem. 80, 3058 (2015)
33. Y. Zhou, Y. Xi, J. Zhao, X. Sheng, S. Zhang, H. Zhang, Org. Lett. 14, 3116 (2012)

34. R.B. Labroo, L.A. Cohen, J. Org. Chem. 55, 4901 (1990)

35. W.E. Savige, A. Fontana, Int. J. Pept. Protein Res. 15, 285 (1980) 